\title{
FUNCTIONAL SEQUENTIAL AND TRIGONOMETRIC SUMMABILITY OF REAL AND COMPLEX FUNCTIONS
}

\author{
M.H. HOOSHMAND*
}

\begin{abstract}
Limit summability of functions was introduced as a new approach to extensions of the summation of real and complex functions, and also evaluating antidifferences. Also, limit summand functions generalize the (logarithm of) Gamma-type functions satisfying the functional equation $F(x+1)=f(x) F(x)$. Recently, another approach to the topic entitled analytic summability of functions, has been introduced and studied by the author. Since some functions are neither limit nor analytic summable, several types of summabilities are needed for improving the problem. Here, I introduce and study functional sequential summability of real and complex functions for obtaining multiple approaches to them. We not only show that the analytic summability is a type of functional sequential summability but also obtain trigonometric summability (for functions with a fourier series) as another its type. Hence, we arrive at a class of real and complex function spaces with various properties. Thereafter, we prove several properties of functional sequential, and also many criteria for trigonometric summability. Finally, we state many problems and future directions for the researches.
\end{abstract}

\section{INTRODUCTION}

In the theory of indefinite sum, antidifference and finite calculus, obtaining some special solutions of the difference functional equation

$$
\nabla F(x):=F(x)-F(x-1)=f(x) ; x \in E,
$$

is very important, where $E$ is the domain of a real or complex function $f$ or a subset of $\mathbb{C}$ (analogously for $\triangle F(x):=F(x+1)-F(x)=f(x)$, where $\triangle$ is the forward difference operator, e.g., see [1]).

But the author discovered an approach to the solution of the equation (on 2001) when he tried to generalize the Bohr-Mollerup theorem and Gamma-type functions (see $[2,3,5]$ ). The following are its summary.

Let $f$ be a real or complex function with domain $D_{f} \supseteq \mathbb{N}^{*}=\{1,2,3, \cdots\}$. Put

$$
\Sigma_{f}=\left\{x \mid x+\mathbb{N}^{*} \subseteq D_{f}\right\},
$$

and then for any $x \in \Sigma_{f}$ and $n \in \mathbb{N}^{*}$ set

$$
\begin{gathered}
R_{n}(f, x)=R_{n}(x):=f(n)-f(x+n), \\
f_{\sigma_{n}}(x)=f_{\sigma_{\ell, n}}(x):=x f(n)+\sum_{k=1}^{n} R_{k}(x) .
\end{gathered}
$$

The function $f$ is called limit summable at $x_{0} \in \Sigma_{f}$ if the functional sequence $\left\{f_{\sigma_{n}}(x)\right\}$ is convergent at $x=x_{0}$. The function $f$ is called limit summable on the set $S \subseteq \Sigma_{f}$ if it is limit summable at all the points of $S$.

Now, put

$$
f_{\sigma}(x)=f_{\sigma_{\ell}}(x)=\lim _{n \rightarrow \infty} f_{\sigma_{n}}(x), R(x)=R(f, x)=\lim _{n \rightarrow \infty} R_{n}(f, x) .
$$

Therefore $D_{f_{\sigma}}=\left\{x \in \Sigma_{f} \mid f\right.$ is limit summable at $\left.x\right\}$, and $f_{\sigma_{\ell}}=f_{\sigma}$ is the same limit function of $f_{\sigma_{n}}$ with domain $D_{f_{\sigma}}$.

The function $f$ is called limit summable if it is summable on $\Sigma_{f}, R(1)=0$ and $D_{f} \subseteq D_{f}-1$. In this

Received $17^{\text {th }}$ September, 2017; accepted $21^{\text {st }}$ October, 2017; published $1^{\text {st }}$ November, 2017.

2010 Mathematics Subject Classification. 40A30; 39A10.

Key words and phrases. limit summability; analytic summability; antidifferences. their papers, and all open access articles are distributed under the terms of the Creative Commons Attribution License. 
case the function $f_{\sigma}$ is referred to as the limit summand function of $f$. If $f$ is limit summable, then $D_{f_{\sigma}}=D_{f}-1$ and

$$
f_{\sigma}(x)=f(x)+f_{\sigma}(x-1) ; \forall x \in D_{f} .
$$

Therefore, if $f$ is limit summable then its limit summand function $f_{\sigma}$ satisfies (1.1).

The paper [2] states a framework for studying the limit summable functions including basic conditions, many criteria for limit summability, uniqueness conditions for its summand function and so on.

Example 1.1. If $|a|<1$, then the complex function $f(z)=a^{z}$ is absolutely limit summable and $f_{\sigma}(z)=\frac{a}{a-1}\left(a^{z}-1\right)$. Also, if $0<b \neq 1$ and $0<a<1$, then the real function $g(x)=c a^{x}+\log _{b} x$ is limit summable and

$$
g_{\sigma}(x)=\frac{c a}{a-1}\left(a^{x}-1\right)+\log _{b} \Gamma(x+1) .
$$

One can see the topic of limit summability in [2,3].

Since some of the important special functions are not limit summable, recently, another type of summability entitled "analytic summability" has been introduced in [4]. We obtain it again in this paper (as a type of functional sequential summability).

\section{FunCtional SEQUential SUmmability}

Let $\delta=\left\{\delta_{n}(x)\right\}_{n=0}^{\infty}$ be a linearly independent functional sequence of complex (or real) functions on $E \subseteq \mathbb{C}$. Put

$$
\mathcal{S}_{E}(\delta):=\operatorname{Span}(\delta)=\operatorname{Span}\left(\delta_{0}(x), \delta_{1}(x), \delta_{2}(x), \cdots\right) .
$$

Similarly, if $\delta=\left\{\delta_{n}(x)\right\}_{n=0}^{\infty}$ and $\beta=\left\{\beta_{n}(x)\right\}_{n=0}^{\infty}$ are two functional sequences on $E$ such that $\delta \cup \beta$ is linearly independent, then we may set

$$
\mathcal{S}_{E}(\delta, \beta):=\operatorname{Span}(\delta \cup \beta)
$$

$\left(\mathcal{S}_{E}\left(\delta_{1}, \cdots, \delta_{k}\right)\right.$ can be defined analogously).

Now, let $\delta=\left\{\delta_{n}(x)\right\}_{n=0}^{\infty}$ be as mentioned and $\Delta=\left\{\Delta_{n}(x)\right\}_{n=0}^{\infty}$ a functional sequence satisfying

$$
\Delta_{n}(x)-\Delta_{n}(x-1)=\delta_{n}(x) ; x \in E, n \in \mathbb{N}_{0}=\{0,1,2, \cdots\} .
$$

Since there are infinitely many such functional sequence $\Delta$ (because (1.1) has the general solution $F(x)=F_{p}(x)+\varphi(x)$ where $F_{p}$ is an its solution and $\varphi$ any 1-periodic function), we fix one of them. The equation implies each $\Delta_{n}(x)$ is defined on $E \cup(E-1)$.

Also, since $\Delta$ is linearly independent (see proof of Theorem 2.2), we may put

$$
\mathcal{S}_{E}^{\sigma}(\delta ; \Delta)=\mathcal{S}_{E}^{\sigma}(\delta):=\operatorname{Span}(\Delta)=\operatorname{Span}\left(\Delta_{0}(x), \Delta_{1}(x), \Delta_{2}(x), \cdots\right)
$$

and so $\mathcal{S}_{E}^{\sigma}(\delta)=\mathcal{S}_{E}(\Delta)$. Note that we use the notation $\mathcal{S}_{E}^{\sigma}(\delta)$ when $\Delta$ is well-known in the topic and there is no any risk of confusion.

Example 2.1. If $\delta_{n}(z)=z^{n}$, then $\delta_{n}(z)$ and

$$
\Delta_{n}(z)=\frac{B_{n+1}(z+1)-b_{n+1}}{n+1}
$$

satisfies the conditions, where $B_{n}(z)$ is the Bernoulli polynomial and $b_{n}=B_{n}(1)$ (see [4]). Here $\mathcal{S}_{E}^{\sigma}(\delta)$ is equal to the space of all polynomials with zero constant.

Note. In continuation, we consider $\delta, E, \Delta, \mathcal{S}_{E}(\delta)$ and $\mathcal{S}_{E}^{\sigma}(\delta)$ with the mentioned conditions.

Lemma 2.1. We have $f \in \mathcal{S}_{E}(\delta)$ if and only if there exists a unique $F \in \mathcal{S}_{E}^{\sigma}(\delta)$ satisfying the difference functional equation (1.1).

(Notation. Hence we shall denote $F$ by $f_{\sigma_{\delta}}$, or simply $f_{\sigma}$ when there is no any risk of confusion.)

Proof. If $f \in \mathcal{S}_{E}(\delta)$ then there exist $\delta_{n_{1}}, \cdots, \delta_{n_{r}} \in \delta$ and coefficients $c_{n_{1}}, \cdots, c_{n_{r}}$ such that $f=$ $\sum_{1}^{r} c_{n_{k}} \delta_{n_{k}}$. Putting $F=\sum_{1}^{r} c_{n_{k}} \Delta_{n_{k}}$ we have $F \in \mathcal{S}_{E}^{\sigma}(\delta)$ and $F$ satisfies the equation. Also, if $G \in \mathcal{S}_{E}^{\sigma}(\delta)$ satisfies $(2.1)$ then $G=\sum_{1}^{t} d_{n_{j}} \Delta_{n_{j}}$, for some coefficients $d_{m_{1}}, \cdots, d_{m_{t}}$, and

$$
\sum_{k=1}^{r} c_{n_{k}} \delta_{n_{k}}=F(x)-F(x-1)=f(x)=G(x)-G(x-1)=\sum_{j=1}^{t} d_{m_{j}} \delta_{n_{j}}(x) ; x \in E .
$$


Hence, independence of $\delta$ implies $t=r$ and $\left\{c_{n_{1}}, \cdots, c_{n_{r}}\right\}=\left\{d_{m_{1}}, \cdots, d_{m_{r}}\right\}$. Therefore,

$$
G=\sum_{1}^{r} c_{n_{k}} \Delta_{n_{k}}=F
$$

The converse is obvious (by (2.1)).

Definition 2.1. Let $f(x)=\sum_{0}^{\infty} c_{n} \delta_{n}(x)$ be convergent on $E$. We say the function $f$ is $(\delta, \Delta)$ summable (or simply $\delta$-summable) if the series $\sum_{0}^{\infty} c_{n} \Delta_{n}(x)$ is convergent on $E$ (the terms uniformly and absolutely $\delta$-summable are defined analogously). Moreover, if it is the case, then we may put

$$
f_{\sigma_{\delta}}(x)=f_{\sigma}(x):=\sum_{0}^{\infty} c_{n} \Delta_{n}(x)
$$

and call it $\delta$-summand function of $f$ on $E$ (this symbol agrees with the above notation, i.e., if $f \in \mathcal{S}_{E}(\delta)$ then the function $f_{\sigma}$ that is gotten from Lemma 2.2 and this definition are the same).

Now, we set

$$
\begin{gathered}
\overline{\mathcal{S}}_{E}(\delta):=\{f: E \rightarrow \mathbb{C} \mid f \text { is } \delta \text {-summable }\} \\
\overline{\mathcal{S}}_{E}^{\sigma}(\delta):=\left\{f_{\sigma_{\delta}} \mid f \in \overline{\mathcal{S}}_{E}(\delta)\right\}
\end{gathered}
$$

It is obvious that $\mathcal{S}_{E}(\delta) \subseteq \overline{\mathcal{S}}_{E}(\delta) \subseteq$ the space of all functions with a Fourier series on $E$, and $\mathcal{S}_{E}^{\sigma}(\delta) \subseteq$ $\overline{\mathcal{S}}_{E}^{\sigma}(\delta)$.

Theorem 2.1. The sets $\overline{\mathcal{S}}_{E}(\delta)$ and $\overline{\mathcal{S}}_{E}^{\sigma}(\delta)$ are functions spaces and the map

$$
\sigma=\sigma_{\delta}: \overline{\mathcal{S}}_{E}(\delta) \rightarrow \overline{\mathcal{S}}_{E}^{\sigma}(\delta)
$$

defined by $\sigma(f):=f_{\sigma}$, is a surjective linear map with $\mathcal{S}_{E}^{\sigma}(\delta) \subseteq \sigma\left(\mathcal{S}_{E}(\delta)\right)$. Also, we have

(a) $f_{\sigma}$ satisfies the difference functional equation (1.1).

(b) The surjective linear map $\sigma$ is bijective (and so $\overline{\mathcal{S}}_{E}(\delta) \cong \overline{\mathcal{S}}_{E}^{\sigma}(\delta)$ ) if and only if $\delta=\left\{\delta_{n}(x)\right\}_{n=0}^{\infty}$ is infinitely linearly independent (i.e., $\sum_{0}^{\infty} c_{n} \delta_{n}(x)=0$ on $E$ implies $c_{n}=0$, for all $n \in \mathbb{N}_{0}$, e.g., $\delta_{n}(x)=x^{n}$ or $\left.\delta_{n}(x)=e^{i n x}\right)$. Therefore, if this is the case then $\mathcal{S}_{E}^{\sigma}(\delta)=\sigma\left(\mathcal{S}_{E}(\delta)\right)$.

Proof. It is obvious that $\overline{\mathcal{S}}_{E}(\delta)$ and $\overline{\mathcal{S}}_{E}^{\sigma}(\delta)$ are vector spaces and $\sigma$ is a surjective linear map from $\overline{\mathcal{S}}_{E}(\delta)$ to $\overline{\mathcal{S}}_{E}^{\sigma}(\delta)$. Then, Lemma 2.2 implies $\mathcal{S}_{E}^{\sigma}(\delta) \subseteq \sigma\left(\mathcal{S}_{E}(\delta)\right)$. Now, note that linearly independence (resp. infinitely linearly independence) of $\delta$ on $E$ implies linearly independence (resp. infinitely linearly independence) of $\Delta$ on $E \cup(E-1)$. Because if $\sum c_{n_{j}} \Delta_{n_{j}}(t)=0$ for all $t \in E \cup(E-1)$ then

$$
\sum c_{n_{j}} \Delta_{n_{j}}(x)=0, \sum c_{n_{j}} \Delta_{n_{j}}(x-1)=0 ; \forall x \in E .
$$

Therefore

$$
\sum c_{n_{j}}\left(\Delta_{n_{j}}(x)-\Delta_{n_{j}}(x-1)\right)=\sum c_{n_{j}} \delta_{n_{j}}(x)=0 ; \forall x \in E,
$$

and so all $c_{n_{j}}$ are zero.

Now, if $f, g \in \overline{\mathcal{S}}_{E}(\delta)$ then $f(x)=\sum_{0}^{\infty} c_{n} \delta_{n}(x), g(x)=\sum_{0}^{\infty} d_{n} \delta_{n}(x)$, for all $x \in E$, and $f_{\sigma}(x)=$ $\sum_{0}^{\infty} c_{n} \Delta_{n}(x), g_{\sigma}(x)=\sum_{0}^{\infty} d_{n} \Delta_{n}(x)$, for all $x \in E \cup(E-1)$. So

$$
f_{\sigma}(x)-f_{\sigma}(x-1)=\sum_{0}^{\infty} c_{n}\left(\Delta_{n}(x)-\Delta_{n}(x-1)\right)=\sum_{0}^{\infty} c_{n} \delta_{n}(x)=f(x) ; \forall x \in E .
$$

Also, if $\sigma(f)=\sigma(g)$, then $\sum_{0}^{\infty}\left(c_{n}-d_{n}\right) \Delta_{n}(x)=0$ on $E \cup(E-1)$ and so $c_{n}-d_{n}=0$ (because $\delta$ is infinitely linearly independent), which means $\sigma$ is injective. Hence the proof is complete. 
2.1. Analytic summability. Let $\delta_{n}(z)=z^{n}(z \in \mathbb{C})$ and consider all terms of Example 2.1 on an open domain $D=E \subseteq \mathbb{C}$. Then $\overline{\mathcal{S}}_{D}(\delta)$ is a subspace of all analytic functions on $D$. Also, $f(z)=\sum_{0}^{\infty} c_{n} z^{n} \in \overline{\mathcal{S}}_{D}(\delta)$ is $\delta$-summable if and only if the series

$$
\sum_{0}^{\infty} c_{n} \frac{B_{n+1}(z+1)-b_{n+1}}{n+1}
$$

is convergent on $D$, and this is the same "Analytic summability of $f$ " from [4]. Therefore (according to [4]) we denote it by $f_{\sigma_{\mathcal{A}}}$ instead of $f_{\sigma_{\delta}}$ and call this $f$ "Analytic summable", and also denote the space by $\overline{\mathcal{S}}_{D}(\mathcal{A})$. Theorem 2.4 implies $\sigma_{\mathcal{A}}$ is a surjective linear map from $\overline{\mathcal{S}}_{D}(\mathcal{A})$ to $\overline{\mathcal{S}}_{D}^{\sigma}(\mathcal{A})$, and so $\overline{\mathcal{S}}_{D}(\mathcal{A}) \cong \overline{\mathcal{S}}_{D}^{\sigma}(\mathcal{A})$ that is a new result about analytic summability. One can see many criteria, results and open problems about this type of functional sequential summability in it.

Example 2.2. The exp function is analytic summable in $\mathbb{C}$ and

$$
\begin{gathered}
\exp _{\sigma}(z)=\sum_{n=0}^{\infty} \frac{1}{n !} \sigma\left(z^{n}\right)=\lim _{N \rightarrow \infty} \sum_{n=0}^{N} \sum_{k=1}^{n+1} \frac{1}{n !} \frac{n !}{k !(n+1-k) !} b_{n+1-k} z^{k} \\
=\lim _{N \rightarrow \infty} \sum_{n=1}^{N+1} \sum_{k=n-1}^{N} \frac{1}{n !} \frac{b_{k+1-n}}{(k+1-n) !} z^{n}=\sum_{n=1}^{\infty} \frac{1}{n !}\left(\sum_{j=0}^{\infty} \frac{b_{j}}{j !}\right) z^{n} \\
=\frac{e}{e-1} \sum_{n=1}^{\infty} \frac{z^{n}}{n !}=\frac{e}{e-1}\left(e^{z}-1\right) .
\end{gathered}
$$

So $\exp _{\sigma_{\mathcal{A}}}(z)=\frac{e}{e-1}\left(e^{z}-1\right)$ (details can bee seen in [4]).

\section{Trigonometric SUmmability}

Now, we can introduce another important type of functional sequential summability in two ways: (a) Consider $\gamma=\left\{e^{i n x}\right\}_{n=-\infty}^{\infty}(x \in \mathbb{R}, n \in \mathbb{Z})$. Here, the indices set $\mathbb{N}_{0}$ is replaced by $\mathbb{Z}$. Therefore, in the definition of functional sequential summability, $f$ has the Fourier series form $f(x)=\sum_{-\infty}^{\infty} c_{n} e^{i n x}$. Putting $\Gamma_{n}(x)=\frac{e^{i n}}{e^{i n}-1}\left(e^{i n x}-1\right)$ for $n \neq 0$ and $\Gamma_{0}(x)=c_{0} x$, one see that $\Gamma_{n}$ satisfies the conditions (for all real numbers $x$ ). Hence, $f$ is $\gamma$-summable on $E$ if and only if the following series is convergent

$$
\sum_{-\infty}^{\infty} c_{n} \Gamma_{n}(x) ; x \in E
$$

(b) Put $\delta=\{\cos (n x)\}_{n=0}^{\infty}$ and $\beta=\{\sin (n x)\}_{n=0}^{\infty}(x \in \mathbb{R})$. The sequence $\delta \cup \beta$ is infinitely linearly independent. For every positive integer $n$, put

and also $\Delta_{0}(x)=1, \mathfrak{B}_{0}(x)=0$.

$$
\begin{gathered}
\Delta_{n}(x)=\frac{\cos (n x)+\cos (n)-\cos (n x+n)-1}{2(1-\cos (n))}, \\
\mathfrak{B}_{n}(x)=\frac{\sin (n x)+\sin (n)-\sin (n x+n)}{2(1-\cos (n))},
\end{gathered}
$$

It is easy to see that they satisfy the conditions.

It is obvious that $f$ is $\delta, \beta$-summable on $E$ (i.e., $f \in \overline{\mathcal{S}}_{E}(\delta, \beta)$ ) if and only if it has the Fourier series of the form $f(x)=\frac{a_{0}}{2}+\sum_{n=1}^{\infty} a_{n} \cos (n x)+b_{n} \sin (n x)$ and the following series is convergent

$$
\sum_{n=0}^{\infty} a_{n} \Delta_{n}(x)+b_{n} \mathfrak{B}_{n}(x)
$$

Now, let

$$
f(x)=\sum_{-\infty}^{\infty} c_{n} e^{i n x}=\frac{a_{0}}{2}+\sum_{n=1}^{\infty} a_{n} \cos (n x)+b_{n} \sin (n x) .
$$

It is easy to see that the series (3.1) is convergent if and only if (3.2) is convergent, and so we arrive at the following definition. 
Definition 3.1. Let $E, f, \gamma$ and $\delta, \beta$ be as the above. We call $f$ trigonometric summable (on $E$ ) if the series (3.2) (or equivalently (3.1)) is convergent. Also, we put

$$
f_{\sigma_{\text {trg }}}(x)=f_{\sigma}(x):=\frac{a_{0}}{2} x+\sum_{n=1}^{\infty} a_{n} \Delta_{n}(x)+b_{n} \mathfrak{B}_{n}(x)=c_{0} x+\sum_{-\infty}^{\infty} c_{n} \Gamma_{n}(x) ; x \in E,
$$

and call it trigonometric summand of $f$ (on $E$ ).

Now, we prove many equivalent conditions for trigonometric summability and also several criteria for trigonometric summability of functions with a Fourier series.

Theorem 3.1. (Some criteria for trigonometric summability of real and complex functions) Let

$$
f(x)=\frac{a_{0}}{2}+\sum_{n=1}^{\infty} a_{n} \cos (n x)+b_{n} \sin (n x)=\sum_{-\infty}^{\infty} c_{n} e^{i n x}
$$

is defined on $E \subseteq \mathbb{R}$. For $n \neq 0$ put

$$
A_{n}=a_{n}-\cot \left(\frac{n}{2}\right) b_{n}, B_{n}=b_{n}+\cot \left(\frac{n}{2}\right) a_{n}, C_{n}=c_{n}-i \cot \left(\frac{n}{2}\right) c_{n} .
$$

Also, set $A_{0}=a_{0}, B_{0}=C_{0}=0$. Then

(a) The following statements are equivalent:

- $f$ is trigonometric summable on $E$;

- The series $\sum_{n=1}^{\infty} A_{n} \cos (n x)+B_{n} \sin (n x)-A_{n}$ is convergent (to $2 f_{\sigma}(x)-a_{0} x$ ) on $E$;

- The series $\sum_{-\infty}^{\infty} C_{n} e^{i n x}-C_{n}$ is convergent (to $\left.f_{\sigma}(x)-c_{0} x\right)$ on $E$;

- The series $\sum_{n=1}^{\infty} \csc \left(\frac{n}{2}\right) \sin \left(\frac{n x}{2}\right)\left(a_{n} \cos \left(n \frac{x+1}{2}\right)+b_{n} \sin \left(n \frac{x+1}{2}\right)\right.$ ) is convergent (to $\left.f_{\sigma}(x)-\frac{1}{2} a_{0} x\right)$.

(b) If the Fourier series $\sum_{n=1}^{\infty} B_{n} \cos \left(n \frac{x}{2}\right)-A_{n} \sin \left(n \frac{x}{2}\right)$ (i.e., the series is gotten from the Fourier series of $f$, replacing $a_{n}, b_{n}$ and $x$ by $B_{n}, A_{n}$ and $-\frac{x}{2}$ respectively) is absolutely convergent, then $f$ is absolutely trigonometric summable and

$$
\left|f_{\sigma}(x)-\frac{1}{2} a_{0} x\right| \leq \sum_{n=1}^{\infty}\left|B_{n} \cos \left(n \frac{x}{2}\right)-A_{n} \sin \left(n \frac{x}{2}\right)\right| .
$$

(c) If the Fourier series $\sum_{n=1}^{\infty} \frac{a_{n}}{\sin \left(\frac{n}{2}\right)} \cos \left(n \frac{x+1}{2}\right)+\frac{b_{n}}{\sin \left(\frac{n}{2}\right)} \sin \left(n \frac{x+1}{2}\right)$ (i.e., the series is gotten from the Fourier series of $f$, replacing $a_{n}, b_{n}$ and $x$ by $\frac{a_{n}}{\sin \left(\frac{n}{2}\right)}, \frac{b_{n}}{\sin \left(\frac{n}{2}\right)}$ and $\frac{x+1}{2}$ respectively) is absolutely convergent, then $f$ is absolutely trigonometric summable and

$$
\left|f_{\sigma}(x)-\frac{1}{2} a_{0} x\right| \leq \sum_{n=1}^{\infty}\left|\frac{a_{n}}{\sin \left(\frac{n}{2}\right)} \cos \left(n \frac{x+1}{2}\right)+\frac{b_{n}}{\sin \left(\frac{n}{2}\right)} \sin \left(n \frac{x+1}{2}\right)\right| .
$$

Proof. By using the identity $\frac{\sin (n)}{1-\cos (n)}=\cot \left(\frac{n}{2}\right)$, we obtain (for all $n \geq 1$ )

$$
\begin{gathered}
2 \Delta_{n}(x)=\cos (n x)-1+\cot \left(\frac{n}{2}\right) \sin (n x)=2 \sin \left(\frac{n x}{2}\right)\left\{\cot \left(\frac{n}{2}\right) \cos \left(\frac{n x}{2}\right)-\sin \left(\frac{n x}{2}\right)\right\} \\
=\csc \left(\frac{n}{2}\right) \sin \left(n x+\frac{n}{2}\right)-1=2 \csc \left(\frac{n}{2}\right) \sin \left(\frac{n x}{2}\right) \cos \left(\frac{n x+n}{2}\right), \\
2 \mathfrak{B}_{n}(x)=\sin (n x)+\cot \left(\frac{n}{2}\right)(1-\cos (n x))=2 \sin \left(\frac{n x}{2}\right)\left\{\cos \left(\frac{n x}{2}\right)+\cot \left(\frac{n}{2}\right) \sin \left(\frac{n x}{2}\right)\right\} \\
=\cot \left(\frac{n}{2}\right)-\csc \left(\frac{n}{2}\right) \cos \left(n x+\frac{n}{2}\right)=2 \csc \left(\frac{n}{2}\right) \sin \left(\frac{n x}{2}\right) \sin \left(\frac{n x+n}{2}\right), \\
2 \Gamma_{n}(x)=\left(1-i \cot \left(\frac{n}{2}\right)\right)\left(e^{i n x}-1\right) .
\end{gathered}
$$

Therefore,

$$
\begin{gathered}
\sum_{n=1}^{\infty} a_{n} \Delta_{n}(x)+b_{n} \mathfrak{B}_{n}(x)=\sum_{n=1}^{\infty} A_{n} \cos (n x)+B_{n} \sin (n x)-A_{n} \\
=\sum_{-\infty}^{\infty} C_{n}\left(e^{i n x}-1\right)=\sum_{-\infty, n \neq 0}^{\infty} c_{n} \Gamma_{n}(x),
\end{gathered}
$$


and

$$
\begin{gathered}
\sum_{n=1}^{\infty} A_{n}(\cos (n x)-1)+B_{n} \sin (n x)=2 \sum_{n=1}^{\infty} \sin \left(n \frac{x}{2}\right)\left\{B_{n} \cos \left(n \frac{x}{2}\right)-A_{n} \sin \left(n \frac{x}{2}\right)\right\} \\
=\sum_{n=1}^{\infty} \sin \left(\frac{n x}{2}\right)\left(\frac{a_{n}}{\sin \left(\frac{n}{2}\right)} \cos \left(n \frac{x+1}{2}\right)+\frac{b_{n}}{\sin \left(\frac{n}{2}\right)} \sin \left(n \frac{x+1}{2}\right)\right) .
\end{gathered}
$$

Now one can obtain the results, directly.

The above theorem together with the identity $A_{n}^{2}+B_{n}^{2}=\left(a_{n}^{2}+b_{n}^{2}\right) \csc ^{2}\left(\frac{n}{2}\right)$ imply the following corollary.

Corollary 3.1. Let $a_{n}$ and $b_{n}$ are two sequences of real or complex numbers.

(a) If one of the series $\sum_{n=1}^{\infty} \frac{\left|a_{n}\right|+\left|b_{n}\right|}{\left|\sin \left(\frac{n}{2}\right)\right|}, \sum_{n=1}^{\infty} \frac{\sqrt{a_{n}^{2}+b_{n}^{2}}}{\left|\sin \left(\frac{n}{2}\right)\right|}$ is convergent, then the function $f(x):=\frac{a_{0}}{2}+$ $\sum_{n=1}^{\infty} a_{n} \cos (n x)+b_{n} \sin (n x)$ (is defined in $\mathbb{R}$ ) is absolutely and uniformly trigonometric summable on whole $\mathbb{R}$ and

$$
\begin{aligned}
f_{\sigma}(x) & =\frac{1}{2} a_{0} x+\frac{1}{2} \sum_{n=1}^{\infty} A_{n} \cos (n x)+B_{n} \sin (n x)-A_{n} \\
& =\frac{1}{2} a_{0} x+\sum_{n=1}^{\infty} \csc \left(\frac{n}{2}\right) \sin \left(\frac{n x}{2}\right)\left(a_{n} \cos \left(n \frac{x+1}{2}\right)+b_{n} \sin \left(n \frac{x+1}{2}\right)\right) \\
& =\frac{1}{2} a_{0} x+\sum_{n=1}^{\infty} \sin \left(n \frac{x}{2}\right)\left(B_{n} \cos \left(n \frac{x}{2}\right)-A_{n} \sin \left(n \frac{x}{2}\right)\right) \\
& =c_{0} x+\sum_{-\infty}^{\infty} C_{n} e^{i n x}-C_{n}
\end{aligned}
$$

and

$$
f_{\sigma}^{\prime}(x)=\sum_{n=1}^{\infty} n B_{n} \cos (n x)-n A_{n} \sin (n x) .
$$

Hence, $f_{\sigma}^{\prime}(x)$ has a Fourier series in $\mathbb{R}$.

Note. If $\sum_{n=1}^{\infty} a_{n}-\cot \left(\frac{n}{2}\right) b_{n}\left(=\sum_{n=1}^{\infty} A_{n}\right)$ is convergent then trigonometric summability of the function $f(x)=\frac{a_{0}}{2}+\sum_{n=1}^{\infty} a_{n} \cos (n x)+b_{n} \sin (n x)$ is equivalent to convergence of the Fourier series $\sum_{n=1}^{\infty} A_{n} \cos (n x)+B_{n} \sin (n x)$, and so $f_{\sigma}(x)-\frac{1}{2} a_{0} x$ (if exists) has a Fourier series.

Example 3.1. Consider the real function

$$
f(x):=1+\sum_{n=1}^{\infty} \frac{\sin \left(\frac{n}{2}\right)}{n^{2}} \cos (n x)+\frac{\sin \left(\frac{n}{2}\right)}{n^{2}} \sin (n x)
$$

It is defined on whole $\mathbb{R}$ and by using the above results, $f$ is absolutely and uniformly trigonometric summable and

$$
\begin{gathered}
f_{\sigma}(x)=x-\frac{\pi^{2}}{12}+\frac{1}{2} \sum_{n=1}^{\infty}\left(\frac{\csc \left(\frac{n}{2}\right)-\cos \left(\frac{n}{2}\right)}{n^{2}}\right) \cos (n x)+\left(\frac{\cos \left(\frac{n}{2}\right)+\csc \left(\frac{n}{2}\right)}{n^{2}}\right) \sin (n x) \\
=x+\sum_{n=1}^{\infty} \frac{\csc ^{2}\left(\frac{n}{2}\right)}{n^{2}} \sin \left(\frac{n x}{2}\right)\left(\cos \left(n \frac{x+1}{2}\right)+\sin \left(n \frac{x+1}{2}\right)\right) \\
=x+\sum_{-\infty}^{\infty} C_{n} e^{i n x}-C_{n} .
\end{gathered}
$$

Also

$$
f_{\sigma}^{\prime}(x)=1+\sum_{n=1}^{\infty}\left(\frac{\cos \left(\frac{n}{2}\right)+\csc \left(\frac{n}{2}\right)}{n}\right) \cos (n x)+\left(\frac{\cos \left(\frac{n}{2}\right)-\csc \left(\frac{n}{2}\right)}{n}\right) \sin (n x) ; x \in \mathbb{R},
$$

and

$$
\left|f_{\sigma}(x)-x\right| \leq \sum_{n=1}^{\infty} \frac{2}{n^{2}}=\frac{\pi^{2}}{3} \Rightarrow\left|f_{\sigma}(x)\right| \leq \frac{\pi^{2}}{3}+|x| ; x \in \mathbb{R} .
$$


At last, there are some important questions for future studies of trigonometric, analytic and limit summability of functions (similar to the open problems of [4]).

Open problem 1. Let $f$ be an analytic function defined on open domain $D=E$. If $f$ is both analytic and trigonometric summable, then is it true that $f_{\sigma_{\text {trq }}}=f_{\sigma_{\mathcal{A}}}$ on $D$ ?

Open problem 2. Let $f$ be a function with a Fourier series on $E$ with the property $\mathbb{N}^{*} \subseteq E \subseteq \Sigma_{f}$. If $f$ is both limit and trigonometric summable, then is it true that $f_{\sigma_{l}}=f_{\sigma_{\text {trg }}}$ on $E$ ?

Open problem 3. If $f$ is trigonometric summable on $E=D_{f}$, then under what conditions is it a unique solution of the equation (1.1) with the initial property $f_{\sigma}(0)=0$ ? (compare to the uniqueness Theorem 3.1, Corollary 3.4 of [2] and Theorem A, Corollary 3.4 of [3]).

Finally, as another future direction for the researches, one may study and discover other functional sequential summability (by taking some appropriate functional sequences $\delta=\left\{\delta_{n}(x)\right\}_{n=0}^{\infty}$ ), and also intersection of the spaces of limit, trigonometric and analytic summable functions. Note that every constant function $f(x)=c$ lies in the intersection and $\sigma_{\ell}(c)=\sigma_{\mathcal{A}}(c)=\sigma_{r g}(c)=c x$.

\section{REFERENCES}

[1] P.M. Batchelder, Introduction to Linear Difference Equations, Dover Publications Inc., 1968.

[2] M.H. Hooshmand, Limit Summability of Real Functions, Real Anal. Exch. 27(2001), 463-472.

[3] M.H. Hooshmand, Another Look at the Limit Summability of Real Functions, J. Math. Ext. 4(2009), 73-89.

[4] M.H. Hooshmand, Analytic Summability of Real and complex Functions, J. Contemp. Math. Anal., 5(2016), 262-268.

[5] R. J. Webster, Log-Convex Solutions to the Functional Equation $f(x+1)=g(x) f(x)$ : $\Gamma$-Type Functions, J. Math. Anal. Appl., 209 (1997), 605-623.

Young Researchers and Elite Club, Shiraz Branch, Islamic Azad University, Shiraz, Iran

*CORRESPONDING AUTHOR: hadi.hooshmand@gmail.com 